\title{
ON THE BRAUER-SPEISER THEOREM ${ }^{1}$
}

\author{
BY K, L. FIELDS
}

\author{
Communicated by I. N. Herstein, October 1, 1970
}

Let $x$ be an absolutely irreducible rational valued character of a finite group $G$. The component of the group algebra $Q G$ corresponding to $X$ is central simple over $Q$ and the $Q G$-irreducible module of this component affords the character $m_{\mathcal{Q}}(\mathscr{X}) \mathscr{X}$ which is also rational valued; hence this module is isomorphic to its dual, whence its endomorphism ring (i.e. the division algebra appearing in the simple component) is isomorphic to its opposite and so is a quaternion algebra (Albert-Hasse-Brauer-Nöether). This result is known as the Brauer-Speiser Theorem [1], [2].2

We ask: Does every quaternion division algebra central simple over $Q$ appear in some $Q G$ ? The answer is yes: Let $G$ be generated by $x, y, c$ subject to the relations $x^{p}=1$ ( $p$ odd), $y^{-1} x y=x^{a}$ ( $a$ is primitive $\bmod p$ ), $y^{p-1}=c, c^{2}=1$ and $c$ central. Then $Q G$ contains as a simple component the cyclic algebra $\left\langle Q\left(\xi_{p}\right),\langle\tau\rangle,-1\right\rangle$, which is c.s. over $Q$ and has Hasse-invariant $1 / 2$ at $R$ and $p$. The quaternion group of order 8 yields the ordinary quaternion algebra (Hasseinvariant $1 / 2$ at $\boldsymbol{R}$ and 2 ) and so, by taking tensor products, we see that every quaternion algebra is available. ${ }^{3}$

\section{REFERENCES}

1. R. Brauer, Representation theory of finite groups, Lectures on Modern Math., vol. 1, Wiley, New York, 1963. MR 31 \#2314.

2. B. Fein, Note on the Brauer-Speiser theorem, Proc. Amer. Math. Soc. 25 (1970), $620-622$.

University of Chicago, Chicago, Illinois 60637

AMS 1969 subject classifications. Primary 2080; Secondary 1640.

Key words and phrases. Dual, Hasse-invariant.

1 Research supported by The National Science Foundation.

${ }^{2}$ Herstein and the author have shown that a simple component of $Q G$ is descended from the maximal real subfield of its center if and only if it is of index at most 2 in the Brauer group. (To appear in J. Algebra.)

8 This result has also been obtained independently by Mark Benard. 